\title{
Estimation of wake propagation behind the rotors of wind-powered generators
}

\author{
Naumov, I. V.; Mikkelsen, Robert Flemming; Okulov, Valery
}

Published in:

Thermal Engineering

Link to article, DOI:

$10.1134 /$ S0040601516030071

Publication date:

2016

Document Version

Publisher's PDF, also known as Version of record

Link back to DTU Orbit

Citation (APA):

Naumov, I. V., Mikkelsen, R. F., \& Okulov, V. (2016). Estimation of wake propagation behind the rotors of windpowered generators. Thermal Engineering, 63(3), 208-213. https://doi.org/10.1134/S0040601516030071

\section{General rights}

Copyright and moral rights for the publications made accessible in the public portal are retained by the authors and/or other copyright owners and it is a condition of accessing publications that users recognise and abide by the legal requirements associated with these rights.

- Users may download and print one copy of any publication from the public portal for the purpose of private study or research.

- You may not further distribute the material or use it for any profit-making activity or commercial gain

- You may freely distribute the URL identifying the publication in the public portal

If you believe that this document breaches copyright please contact us providing details, and we will remove access to the work immediately and investigate your claim. 


\title{
Estimation of Wake Propagation behind the Rotors of Wind-Powered Generators
}

\author{
I. V. Naumov ${ }^{a}$, R. F. Mikkelsen ${ }^{b}$, and V. L. Okulov ${ }^{a}$ \\ ${ }^{a}$ Kutateladze Institute of Thermophysics, Siberian Branch, Russian Academy of Sciences, \\ pr. Akademika Lavrent'eva 1, Novosibirsk, 630090 Russia \\ ${ }^{b}$ Technical University of Denmark, Department of Wind Energy, Nils Koppels Alle 403, Lyngby, DK-2800 Denmark \\ e-mail: naumov@itp.nsc.ru
}

\begin{abstract}
The objectives of this work are to develop the experimental model of wake behind the wind-power generator rotor to estimate its propagation distance and the impact on the average and pulsation characteristics of incident flow with the possibility of further use of these data in the calculation models of wind and climate changes in the regions and to determine the optimal operation of wind turbines. For experimental modeling, the laboratory model of wind-powered generator with a horizontal axis was used that operated as wind turbine in optimal mode. The kinematic characteristics of flow and changes in the wake structure in the distance of more than 40 rotor diameters downstream with a slight level of turbulent pulsations (less than 2\%) of free flow were investigated. A significant impact of external intense pulsations typical for natural atmospheric conditions was purposely excluded in the experimental research in order to define the degree of self-damping of perturbations generated by oneself wind-powered generator. The obtained experimental data for the wake dynamics behind the model of wind-powered generator allowed ascertaining its impact on slowing down of incident vortex flow and determining the distance at which its impact on the stream disappears, and the deceleration values are comparable to the level of pulsations of incident flow. This experimental model with the same degree of damping its velocity and pulsations can be used to adjust the theoretical approximation of the far wake. It is shown that the recovery of velocity of incident flow is faster than has been previously defined in the models of calculating the impact of wind electric power plants on the regional climate changes. Thus, existing wind loss calculated on the model of wake behind the wind-powered generator, adjusted in this study can be even less significant.
\end{abstract}

Keywords: wind-power engineering, wake behind the wind turbine rotor, velocity deficit, laser Doppler anemometry (LDA)

DOI: $10.1134 / \mathrm{S} 0040601516030071$

Rotating rotors of wind-powered generators take part of the kinetic energy of the moving air masses, which greatly reduces the wind speed and causes the intensive pulsations in wakes behind them. It can be assumed that this slowing down could have a significant influence on climatic conditions of the area during large-scale use of wind-powered generators. This fact was even attempted using [1] to justify increasing the temperature at $0.72^{\circ} \mathrm{C}$ found for nine years of surveillances over a vast territory in the United States, densely built-up with wind-power engineering plants. If this fact is confirmed, then one can talk about the negative effect of wind-power engineering, i.e., the possibility of global warming as a result of the work of wind electric power plants, which is traditionally considered a "green" energy source. In [1], such correlation was not evidently established, i.e., the found effect could be associated with other impacts on the environment in the area. On the contrary, the recent calculations did not confirm the negative impact of wind electric power plants on the weather [2]. The authors of [2] designed the climate model and calculated the climate changes in the countries of the European Union without taking into account the influence of wind parks installed on their territory. According to these calculations, the temperature in the location zone of wind parks can periodically change slightly, maximum increasing in winter just by $0.1^{\circ} \mathrm{C}$ and going back to the initial value in the summer months. This very insignificant factor should not lead to global negative consequences. It should be noted that the latter result was obtained with rough modeling of the impact of wind-powered generators on the change of wind speed. The authors calculated the wind losses entering in the plane of rotors of windpowered generators energy take-off according to maximum power coefficient of wind turbines, equal to the Betz-Joukowsky limit [3]. This limit is based on an idealized model for value of absolute maximum of wind energy losses in a fixed plane of the rotor and has 
substantially inflated values compared to the real work of the wind-powered generator. In addition, taking into account the energy take-off of flow only in the plane of rotors of a wind-powered generator during calculations can provide only the first rough estimation of their impact on the incident flow, since this approach does not reflect the distance of propagation and intensity of the impact of pulsations in the wakes behind rotors. Thus, it is necessary to have more precise information on the formation and dynamics of wakes behind the rotors of wind-powered generators.

For the basis of these studies, we used the laboratory model of a wind-powered generator that was proposed to study the middle wake behind the rotor in the water channel [4-6]. The laboratory model of a rotor with a horizontal axis and profile for blades CD7003 was designed for optimal calculation speed parameter $\lambda=5\left(\lambda=\Omega R / u_{0}\right.$, where $R$ is the rotor radius, $\Omega$ is the angular velocity of rotor rotation, and $u_{0}$ is the velocity of free flow). The research of three-bladed rotor in radius $R=0.188 \mathrm{~m}$ (Fig. 1) was continued in this work. It was flowed around with constant flow at speed $u_{0}=$ $0.61 \mathrm{~m} / \mathrm{s}$ and pulsation level of incident flow less than $2.5 \%$ [4]. The significant impact of external intense pulsations typical for natural atmospheric conditions was deliberately excluded in the experimental studies. This was done to identify the influence of rotor wake on the incident flow up to determination of the distance, where its impact on the flow disappears, and the values of velocity deficiency are comparable with the level of their own pulsations of incident flow. The dimensions of working section of water channel in $30 \mathrm{~m}$ length and cross-section $3 \times 1 \mathrm{~m}$ at the central position of the rotor allowed avoiding interaction of the wake with boundary layer (whose thickness is less than $0.2 \mathrm{~m}$ ) of bottom and sides of the channel, which is controlled in accordance with the work [4]. The rotor was located on the platform moving over the channel at a distance at $0.3 \mathrm{~m}$ behind it to minimize the disturbance of incident flow in the rotor plane. The rotor axis was located at a height at $0.5 \mathrm{~m}$ of the bottom, to minimize the impact of heterogeneity of the incident flow profile. The servomotor JVL Industry Elektronik MAC400 was applied to rotate the rotor with constant angular velocity. The motor torque was passed to rotor axle with gearing. The control software of servomotor provided the required angular velocity of rotor rotation and monitoring with an error of less than $1.5 \%$.

Behind the rotor operating as wind turbine with $\lambda=5$, the dynamics of flow and changing the structure of the wake over a distance more than 40 rotor diameters $(D)$ were investigated. For the first time, this was carried out with such low turbulence of incident flow and on such large relative distance behind the rotor to establish the degree of self-damping of perturbations generated with the wind-powered generator itself. (a)

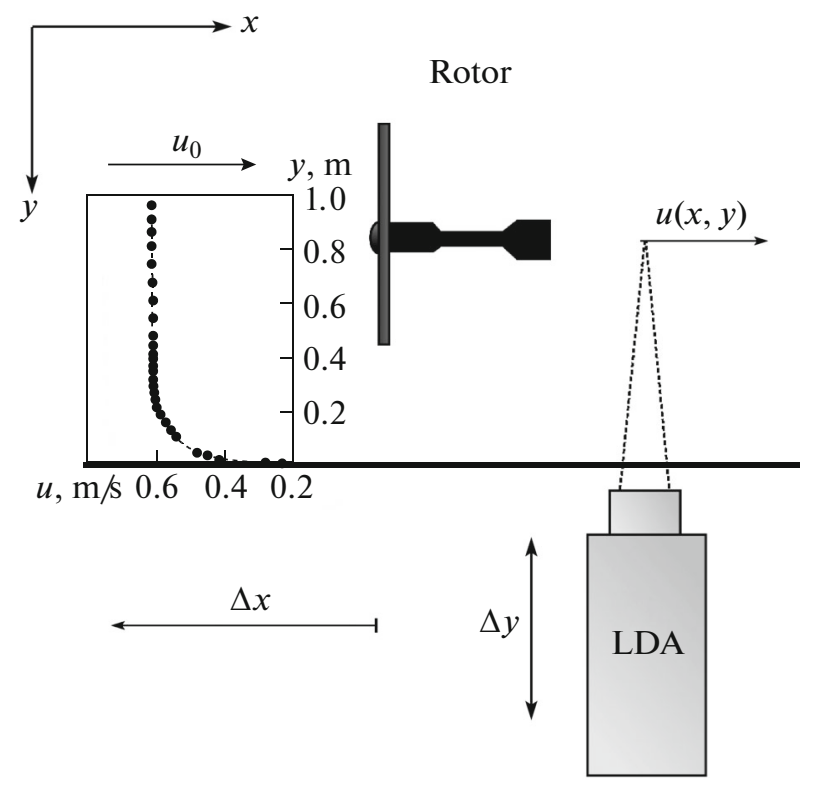

(b)

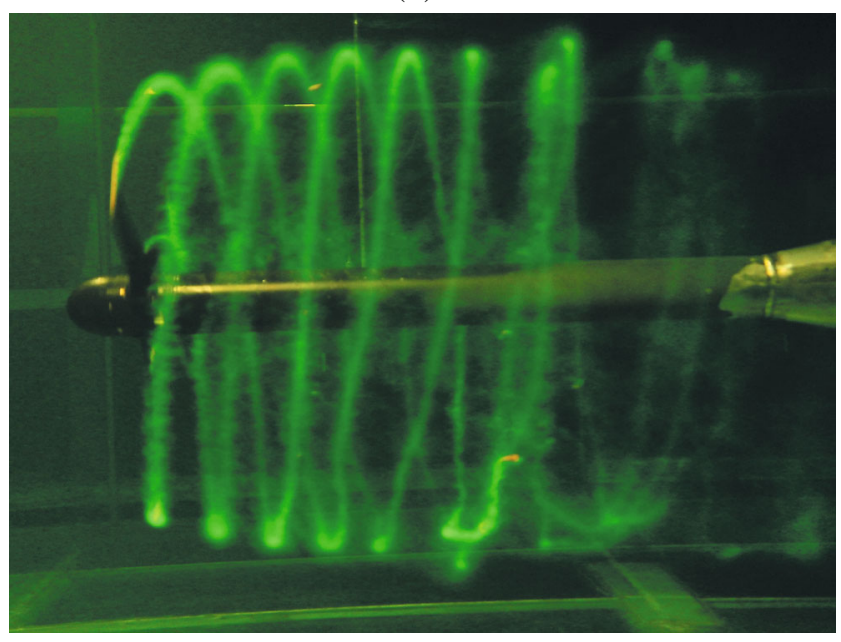

Fig. 1. (a) Experimental diagram and (b) visualization of the near wake [7].

To determine the coefficient of energy utilization of incident flow, the tensometric measurements of torque and the load on the rotor at different velocity [4] were carried out. The voltage from the tensometric sensors was amplified with preamplifier Scout 55 produced by Hootinger Baldwin Messtechnik GmbH and recorded with the system of National Instruments Co., Inc., for collecting the data from sensors with a frequency of $100 \mathrm{~Hz}$ during $60 \mathrm{~s}$. With the received data, the power and drag coefficients were calculated. In the experimental studies, the real coefficient of utilization of the kinetic energy of the incident flow does not exceed $40 \%$, whereas if we introduce the BetzJoukowsky limit [2] into the calculation, this coefficient is $59.3 \%$, i.e., almost 1.5 times more. This ideal- 


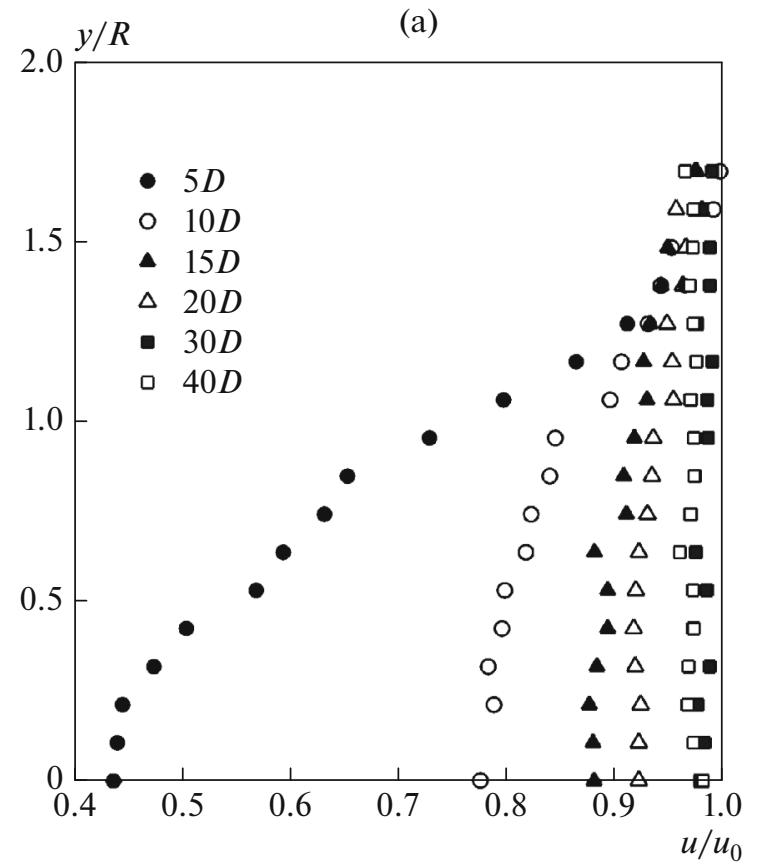

(b)

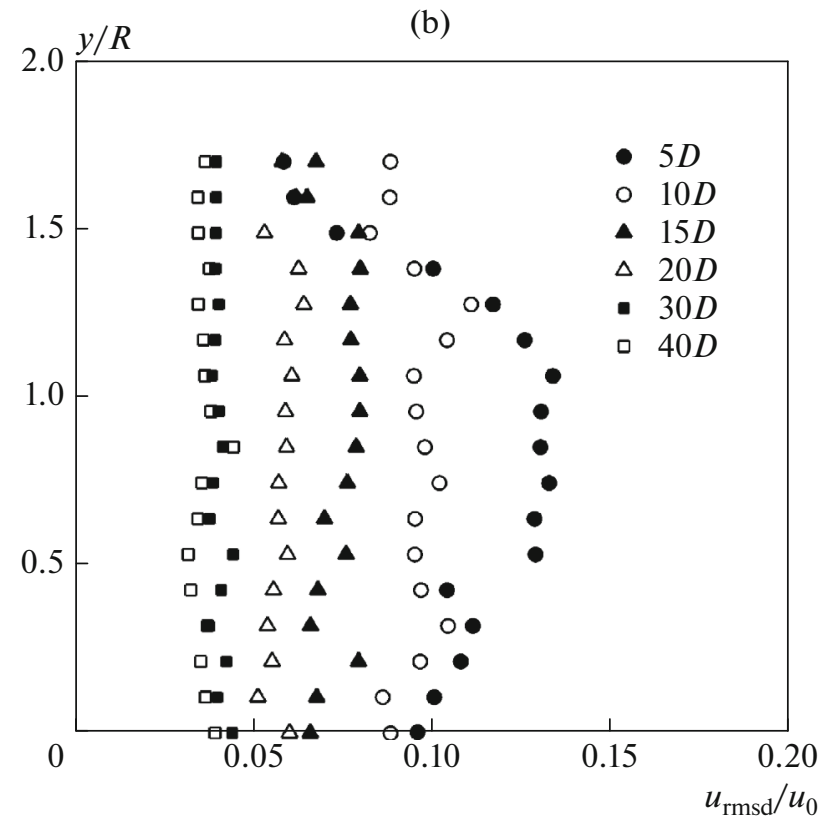

Fig. 2. Average profiles of (a) axial velocity and the (b) root-mean-square of velocity in different sections of the wake for the model of a three-blade rotor.

ized limit is not very accurate even in comparison with the calculation for other ideal rotor models $[8,9]$. At optimal tip speed ratio $\lambda=5$, the calculation on these models gives the value of coefficient equal to $49.7 \%$, i.e., closer to the experimental one than at using the Betz-Joukowsky limit. In addition to this, the energy take-off on the rotor occurs at reducing the velocity of incident flow only by one third, while the maximum velocity of flow slowing down in the wake continues to grow to two-thirds at the distance at (3-4) $R$ and only then increasing slowing down of motion in the wake begins $[5,6]$ to its complete disappearance.

It should be noted that all existing ideal theories of rotor do not allow taking into account the influence of unsteady pulsations in the wake [6]. They also cannot answer the yet unstudied question on the possibility of full damping of perturbations and pulsations generated by the rotor in a uniform incident flow. It is clear that, in different climate models, including the quoted model [2], to obtain more correct calculation results, it is necessary to accurately estimate the impact of wind-powered generator on the change of initial wind velocity, growth and damping of pulsations generated by them, and refined wake length, including that in this experiment.

The study of the wake structure behind the rotor always was carried out in one section of the channel, and the distance in the wake up to the rotor was changed by moving it to a preset position ( $\Delta x$, see Fig. 1a) upstream along the channel centerline. In the course of research in the same testing channel section, the same effect of boundary layer from its walls to the wake zone behind the rotor was ensured. The rotor at its motion upstream on the flow was always in the zone of flowing around with uniform incident flow, because the thickness of the boundary layer with the walls at the beginning of the channel has almost two times lesser value and does not affect the flow profile in the middle of the channel, where the model was placed.

To measure the velocity components and their pulsations, the two-component laser Doppler anemometer (Dantec 2-D LDA) was used. Figure 1a shows its location in the experiment. The anemometer was realized on the argon laser of $2 \mathrm{~W}$ power and commercial processor BSA57N2 produced by Dantec Dynamics to processing the Doppler signals. In this LDA, the differential dual-frequency optical configuration was applied (the shift frequency is $40 \mathrm{MHz}$ ). The diameter of the optical head is $112 \mathrm{~mm}$, the focal length is $700 \mathrm{~mm}$, and the diameter of laser beams is $1.35 \mathrm{~mm}$. The wavelengths of laser emission are 514.5 and $488 \mathrm{~nm}$, and the dimensions of the measuring zone of LDA is $0.12 \times 0.12 \times 1.52 \mathrm{~mm}$. The displacement of measuring zone across the channel ( $\Delta y$, see Fig. 1a) was carried out to restore the full velocity profile in different sections behind the rotor model of the windpowered generator. The duration of the Doppler signal accumulation was $100 \mathrm{~s}$ at the quantity of recorder Doppler flashes about 80 per second. This provided a sample of recorded events more than 6000 . Then, the signal processor processed the Doppler signal from the light-scattering particles.

The general representation of the flow structure behind the rotor operating in turbine mode has been known for a long time. Taking-off the incident flow energy, the turbine rotor brakes it, and the typical dip of velocity is formed in the wake (Fig. 2a). On the ideal theory of a rotor with an infinite number of blades [3] 
used in [2], this braking action in the plane of the rotor operating in optimal mode is $1 / 3$ of the incident flow speed. According to this theory, the further growth of slowing down the flow velocity in the wake up to a value of $2 / 3$ of unperturbed velocity at the distance from rotor to infinity is expected. Behind the real three-blade rotor, this decreasing the flow velocity are recorded at all measurements in the near wake $[5,6]$. Its appearance here, when the effect of the turbine to the flow was ended, is defined by the system of helical vortexes, coming down off the ends of the blades (Fig. 1b) and generating additional slowing down the flow. However, in contrast to the prediction of the ideal models $[3,8,9]$ this growth of slowing down in the wake does not continue indefinitely. This is due to the absolute instability of helical vortex system [7, 10, 11]. At a distance of (2-4) $D$ behind the rotor, the helical vortices are destroyed, and their effect on the flow stops. After the destruction of the near wake and achievement of maximum braking at distance of $(2-5) D$ of the rotor the forming far wake begins [4]. In fact, its structure and properties differ from those at the fixed form of helical vortexes of the near wake accepted in ideal models $[8,9]$ and preserving its deterministic blade configuration of the moment of the coming-off from rear edges to infinity. In fact, it occurs here forming another wake that does not depend on the blade structure of near wake of the rotor and the periodicity of its rotation. It becomes like a wake behind the motionless blunt-nosed body-a disc or sphere [4]. The braking zone of vortex wake swirled in a large-scale helical spiral, and, as a result of its low frequency oscillations, the wake is eroded far behind the rotor: slowing down disappears and the profile of initial incident flow is fully restored.

To illustrate the above, analyze the change of the profile of the average axial velocity in the far wake behind the rotor depending on the distance from it. The profiles were measured in a radial direction from the rotor centerline (it is the line passing through the rotor axis up to distance of $1.8 R$ from it; see Fig. 2a). After the complete formation of the far wake at a distance of $5 D$, the speed profile in the wake has the maximum braking, which decreases rapidly at the further increase in the distance from the rotor and almost completely disappears after $30 \mathrm{D}$.

To clearly estimate the change of flow velocity damping in the wake, Fig. 3a represents the changes of axial velocity at increasing the distance from the rotor along a central axis. First, these data confirm the growth of braking in the near wake from $x=0$ to $x=5 D$. The growth of braking stops a little before, which, apparently, is connected with the destruction of the system of end vortices. The beginning of their destruction is visually surveyed when $x=D$ (see Fig. 1b). At points of far wake $(x>5 D)$, the damping of braking has a monotonic character; furthermore, the change of its speed submits to a power law with an exponent found for the damping of wake behind a flat disc [12, 13]. For
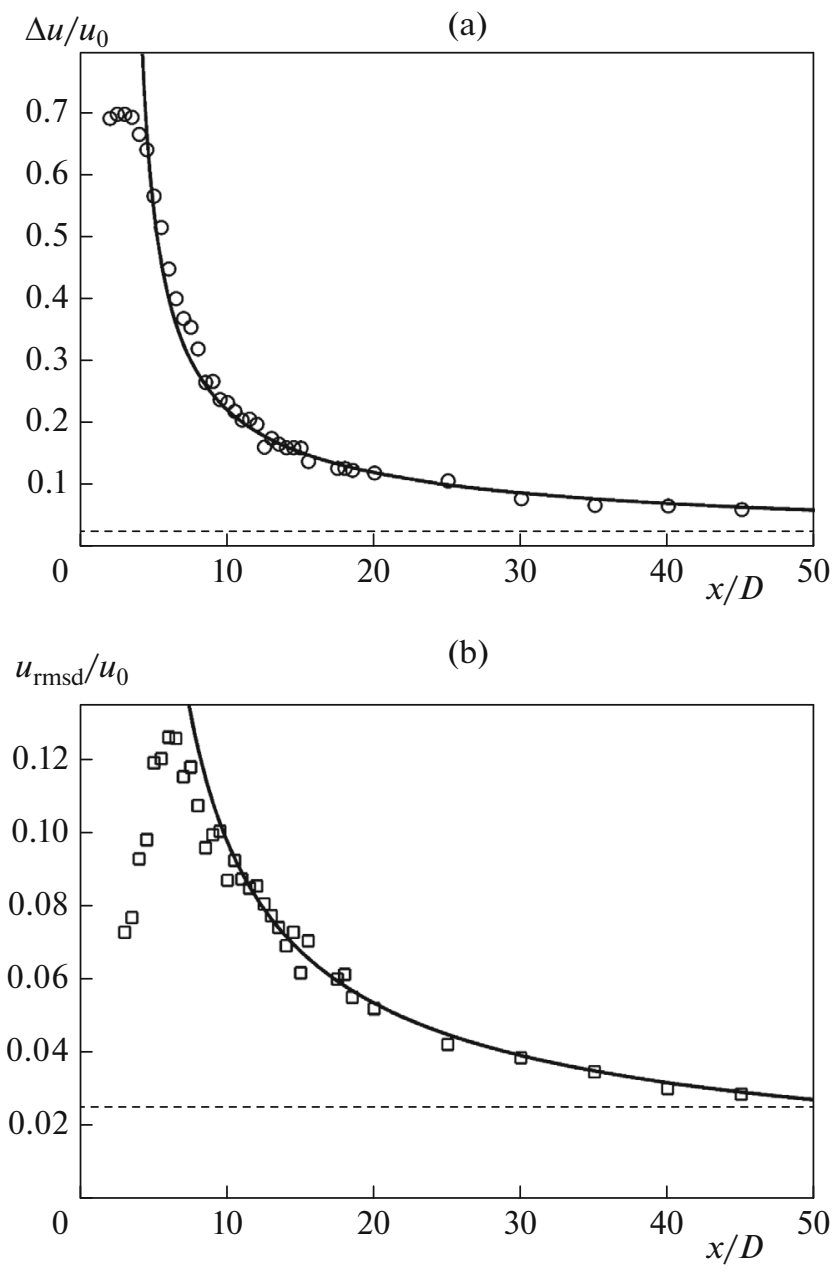

Fig. 3. Damping of (a) velocity braking and (b) standard deviation behind the model of a three-blade rotor. The solid line is the approximation with degree $(-2 / 3)$ and the dotted line is the level of natural disturbances in hydro channel $(2.5 \%)$.

damping of velocity in the wake behind the rotor, one can write a formula

$$
\frac{\Delta u}{u_{0}}=\frac{\max \left[u_{0}-u(x, y)\right]}{u_{0}}=a\left(x-x_{0}\right)^{-\frac{2}{3}},
$$

where $u_{0}$ is the velocity of free flow; $u(x, y)$ is the longitudinal component of speed in a cross section of the wake behind the rotor; $a=0.80$ and $x_{0}=3.2$, which differs from the values defined in $[12,13]$ for damping of the wake behind a thin disk: $a=0.31$ and $x_{0}=3.2$.

It should be noted that, if we exclude the piece of the near wake, that braking the far wake behind the rotor submits to the law of damping (1) along the whole length of its testing from $x=5 D$ to $x=50 D$ (Fig. 3a shows the comparison of continuous curve with the experimental values marked by the symbols).

To determine the values of the parameters of pulsation velocity in the wake, the root-mean-square (RMS) 
of velocity in the wake at different distances from the rotor were found. Figure $2 b$ presents the RMS profiles in the different sections of the wake and Fig. 3b shows the degree of damping of maximum velocity pulsations along the whole length of measured wake. These data indicate that, from the beginning, the growth of speed pulsation in the near wake is surveyed and at the initial piece of formation of far wake from $x=0$ to $x=5 D$; then, the damping in the far wake at $x \geqslant 5 D$ occurs. It should be pointed out that pulsation damping of flow velocity in the points of the far wake is submitted to the same power law that velocity braking damping and (1), but at slightly different meaning of factor $a$, i.e.,

$$
\frac{\max \left[U_{\mathrm{SD}}(x, y)\right]}{u_{0}}=b\left(x-x_{0}\right)^{-\frac{2}{3}}
$$

where $U_{\mathrm{SD}}(x, y)$ is the longitudinal component of the RMS in cross-section of the wake behind the rotor; $b=0.35$, and $x_{0}=3.2$ retains its value.

According to the damping diagram in Fig. 2b, the values of velocity pulsations in the wake in the last measured cross section $(x=5 D)$ at the level of $2.5 \%$ coincided with the background values of velocity pulsations of incident flow in the channel, i.e., then the wake pulsations becomes unrecognizable. This practically means that pulsations formed by the wake have value only when they exceed the pulsations of incident flow.

To completely describe the structure of pulsation dynamics in the wake, it is necessary to indicate the dominant values of frequencies. They were defined in [4] and confirmed by experiments carried out by the authors of this article. In the near wake, the rotational speed of the rotor and its blade rate dominate; in the far wake (when $x \geqslant 3 \mathrm{D}$ ), the low frequency of oscillations dominates, and the value is determined by the Strouhal number calculated on the rotor diameter and the velocity of incident flow and is equal to 0.23 .

Different environmental problems of wind-power engineering were examined in the works $[14,15]$. The authors of the works analyzed the possible effects of wind-powered plants (WPP) on man and the environment by the example of 60 MWWPP in the Mirny village of Yeysk district of Krasnodar region [16] and Far-Eastern 32 MW WPP in Primorskii krai. The authors carried out the integrated assessment of the negative influence of the WPP on the main components of the natural environment: air, water, soil, vegetation, and fauna. Basically, this effect proved to be negligible compared to the influence of traditional energy sources. Regarding pulsations generated by rotor wakes, the authors noted even some positive impact of extra turbulence of flow behind the wind turbines that was in improving the weather conditions for agriculture. In other words, if the negative effect of the weak impact of this additional turbulence on global temperature rise is currently disputable and only discussed [1, 2], then its local positive effect was already pointed out in works [14, 15].

Thus, in this study, the authors experimentally simulated for the first time the full structure of the dynamics of far wake behind the rotor operating in the wind turbine mode at optimal speed. This allowed us to detect the degree of self-damping of average value of velocity braking and pulsations in the wake and define the border of their disappearance. The proposed experimental model with the same degree of speed braking damping (1) and its pulsations (2) can be used to refine the theoretical approximation of the far wake. It should be pointed out that the more complete study of the far wake at the distance up to 50 rotor diameters allowed us to define more precisely the degree of damping of its wake than previously in work [17] when studying the short pieces of the wake not exceeding 10 rotor diameters in length. In the future, the application of the proposed experimental models (1) and (2) and refined theoretical model $[8,9]$ will make the calculations on various climate models more accurate than the used, as in [2], inflated values of rotor power specifications calculated according to the Betz-Joukowsky limit [3].

Presented experimental data on damping the braking velocity and its pulsations in the wake can apply to refine the assessment of the impact of real intense pulsations of atmospheric boundary layer as the more powerful factor of destruction of wake behind a windpowered generator. Such assessed calculations are carried out to optimize the location of wind-powered generators in the parks of wind-powered plants. According to experimental modeling implemented in this work, the distance for self-damping the wake up to the level of units percent and restoring the initial incident on the rotor flow at recalculation to the blade dimensions of modern turbine up to $80 \mathrm{~m}$ is more than $3 \mathrm{~km}$. In the real wind park, the distance between adjacent turbines is in ten times less. In this case, the effect of the earlier destruction of wakes due to intense external pulsations of turbulent atmospheric boundary layer is taken into account. The calculation of influence of external pulsation of different intensity on the initial unperturbed wake was carried out, for example, in the work [18]. However, the authors in the initial model usually do not take into account the possibility of self-damping the wake (1) and its pulsations (2) found in this study, and determine the impact of turbulent atmospheric boundary layer on the initial nondumping model of the wake. It is, therefore, expected that, to optimize the operation of wind-powered generators, the experimental model of dynamics of selfdamping wake behind the rotor proposed in this article will allow obtaining more reliable data for the calculation of the wakes behind the real wind-powered generators. In addition, it enables to optimize more accurately the operation of wind-powered generators in the parks of wind-powered plants. 
In conclusion, it should be once again emphasized that, in this paper, we speak only about the possible very low influence of wind-powered generators on the change of regional climatic conditions, whose positive $[14,15]$ or negative [1] influence, because of the combined lack of it [2], is still unclear. Of course, this cannot be compatible with global negative effects for burning of organic or nuclear fuels in traditional power plants, the fight against which today stimulates rapid development of "green" energy based on renewable energy sources [19]. This article does not discuss the complex environmental challenges of global energy, but it solves the very specific task of right simulation of the wakes behind the wind-powered generators to accurately assess the degree of damping of incident wind flow. The proposed experimental model will help to more precisely solve the above-listed local tasks concerning possible regional climatic changes and optimization of the wind-powered plants operation.

\section{ACKNOWLEDGMENTS}

The study was implemented with the financial support of the Russian Science Foundation (project no. 14-19-00487).

\section{REFERENCES}

1. L. Zhou, Yu. Tian, S. B. Roy, C. Thorncroft, L. F. Bosart, and $\mathrm{Yu} \mathrm{Hu}$, "Impacts of wind farms on land surface temperature," Nature Climate Change 2, 539-543 (2012).

2. R. Vautard, F. Thais, F. Bréon, I. Tobin, J.-G. D. Lavergne, A. Colette, P. Yiou, and P. M. Ruti, "Regional climate model simulations indicate limited climatic impacts by operational and planned European wind farms," Nature Commun. 5, 1-9 (2014).

3. V. L. Okulov and G. A. M. Kuik, "The Betz-Joukowsky limit: On the contribution to rotor aerodynamics by the British, German and Russian scientific schools," Wind Energy 15 (2), 335-344 (2012).

4. V. L. Okulov, I. V. Naumov, R. F. Mikkelsen, I. K. Kabardin, and J. N. Sorensen, "A regular Strouhal number for large-scale instability in the far wake of a rotor," J. Fluid Mech. 747, 369-380 (2014).

5. I. V. Naumov, V. V. Rakhmanov, V. L. Okulov, K. M. Velte, K. E. Maier, R. F. Mikkel'sen, "Flow diagnostics downstream of a tribladed rotor model," Thermophys. Aeromechanics 19, 171-181 (2012).

6. I. V. Naumov, R. F. Mikkelsen, V. L. Okulov, and J. N. Sørensen, "PIV and LDA measurements of the wake behind a wind turbine model," J. Phys.: Conf. Ser. 524, 1-10 (2014).
7. J. H. Walther, M. Guénot, E. Machefaux, J. T. Rasmussen, P. Chatelain, V. L. Okulov, J. N. Sørensen, M. Bergdorf, and P. Koumoutsakos, "A numerical study of the stability of helical vortices using vortex methods," J. Phys.: Conf. Ser. 75, 1-16 (2007).

8. V. L. Okulov and J. N. Sorensen, "An ideal wind turbine with a finite number of blades," Doklady Phys. 53 (6), 337-342 (2008).

9. V. L. Okulov, "Testing of rotor vortex theories using Betz optimization," Doklady Phys. 59(1), 16-20 (2014).

10. V. L. Okulov and J. N. Sørensen, "Instability of vortex wakes behind wind turbines," Doklady Phys. 49 (12), 772-776 (2004).

11. V. L. Okulov and J. N. Sørensen, "Applications of 2D helical vortex dynamics," Theor. Compt. Fluid Dyn. 24, 395-401 (2010).

12. P. B. Johansson, W. K. George, and M. J. Gourlay, "Equilibrium similarity, effects of initial conditions and local Reynolds number on the axisymmetric wake," Phys. Fluids 15, 603-617 (2003).

13. P. B. Johansson and W. K. George, "The far downstream evolution of the high-Reynolds number axisymmetric wake behind a disk. Part 1. Single-point statistics,” J. Fluid Mech. 555, 363-385 (2006).

14. M. A. Ryzhenkov, B. V. Ermolenko, and G. V. Ermolenko, "Environmental problems of wind power engineering," Therm. Eng. 58 (11), 962-969 (2011).

15. B. V. Ermolenko, G. V. Ermolenko, and M. A. Ryzhenkov, "Windenergetics and environmental medium," Energiya: Ekonomika, Tekhnika, Ekologiya, No. 8, 10-19 (2011).

16. G. V. Ermolenko, I. G. Gordeev, A. V. Nikomarova, M. A. Ryzhenkov, and V. N. Tskhomariya, "Pilot projects of network wind power in the Eisk region of Krasnodar krai: The state and prospects," Therm. Eng. 59 (11), 846-853 (2012).

17. M. Bastankhah and F. Porté-Agel, "A new analytical model for wind-turbine wakes," Renew. Energy 70, 116-123 (2014).

18. S.-P. Breton, K. Nilsson, H. Olivares-Espinosa, C. Masson, L. Dufresne, and S. Ivanell, "Study of the influence of imposed turbulence on the asymptotic wake deficit in a very long line of wind turbines," Renew. Energy 70, 153-163 (2014).

19. V. E. Fortov and O. S. Popel', "The current status of the development of renewable energy sources worldwide and in Russia," Therm. Eng. 61 (6), 389-398 (2014). doi 10.1134/S0040363614060022

Translated by M. Kromin 